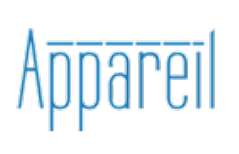

Appareil

$16 \mid 2015$

Individuer Simondon. De la redécouverte aux prolongements

\title{
Penser l'essence de la vie : le matérialisme comme question et comme préalable chez Simondon
}

\section{Émilien Dereclenne}

\section{(2) OpenEdition \\ Journals}

\section{Édition électronique}

URL : http://journals.openedition.org/appareil/2214

DOI : 10.4000/appareil.2214

ISSN : 2101-0714

Éditeur

MSH Paris Nord

Référence électronique

Émilien Dereclenne, «Penser l'essence de la vie : le matérialisme comme question et comme préalable chez Simondon », Appareil [En ligne], 16 | 2015, mis en ligne le 09 février 2016, consulté le 30 juillet 2020. URL : http://journals.openedition.org/appareil/2214 ; DOI : https://doi.org/10.4000/appareil. 2214

Ce document a été généré automatiquement le 30 juillet 2020.

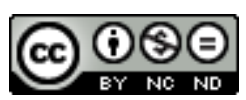

Appareil est mis à disposition selon les termes de la Licence Creative Commons Attribution - Pas d'Utilisation Commerciale - Pas de Modification 4.0 International. 


\title{
Penser l'essence de la vie : le matérialisme comme question et comme préalable chez Simondon
}

\author{
Émilien Dereclenne
}

\section{Introduction}

1 La catégorie de "tropisme ${ }^{1}$ » renvoie aux tendances de l'organisme à rétablir certains équilibres physiologiques. Elle caractérise l'objet propre de la science biologique, à savoir l'organisme que l'on observe dans ou hors de son milieu de vie. Comme Goldstein, Merleau-Ponty y voit l'«essence de la connaissance biologique ${ }^{2} »$. Si la biologie ne se réduit pas à la physique, c'est en effet parce qu'elle étudie le vivant, dont les comportements tropistiques ont une signification biologique que n'ont pas les phénomènes du monde physique. Mais, très simplement et comme y insiste Mikel Dufrenne, si l'on veut éviter de faire de la vie « ce que le corps saisit sur le vivant plutôt que ce qu'il est ${ }^{3}$ ", s'il faut en comprendre l'essence, on ne peut se contenter de l'appréhension des comportements une fois qu'ils sont individués. Il faut s'intéresser aux conditions réelles et concrètes de leur individuation, donc proposer une pensée des genèses (ou ontogenèse). L'objectif est de saisir, au-delà de la signification pour nous d'un être déjà individué, l'en soi d'un être en individuation.

Or, le geste fort de Simondon, dont nous voudrions rendre compte en reprenant le dialogue instructif avec Merleau-Ponty ${ }^{4}$, est de partir d'une réflexion sur la matière pour se donner les moyens conceptuels de penser ce caractère génétique et essentiel du vivant. La thèse principale (1958 $)$ recèle, selon l'expression de Vincent Bontems, un " matérialisme non réductionniste parce qu'énergétiste et néoténique ${ }^{6}$ ", qui rompt avec l'antimatérialisme traditionnel à travers lequel Merleau-Ponty espérait seulement penser la spécificité du vital. Évaluer la portée de ce matérialisme dans l'élaboration d'une pensée non réductionniste de la vie suppose que nous comprenions d'abord comment s'opère cette rupture. Tout l'enjeu est alors de déterminer dans quel sens il 
est effectivement possible de parler de matérialisme à propos de la doctrine de Simondon, malgré ce qu'il en dit lui-même dans sa thèse principale.

\section{Saisir la spécificité du vital}

3 Fidèle au biologisme goldsteinnien, Merleau-Ponty admet d'abord que les structurations vitales du comportement diffèrent des structurations d'un être purement physique. Contre la dérive physicaliste, il s'agit - pour reprendre la formule traditionnelle et épuisée -, de saisir la spécificité du vital par rapport au physique. Mais cette hétérogénéité de principe entre vital et physique ne se pense pas non plus indépendamment d'une certaine forme d'homogénéité. Si les structurations vitales sont d'un genre particulier, elles ne peuvent avoir lieu hors du substrat physique. Il est donc essentiel d'élucider la nature de ce rapport au physique.

4 Merleau-Ponty prétend y parvenir au moyen d'une méthode phénoménologique que nous devons décrypter afin de pouvoir la confronter à celle de Simondon. Cette méthode consiste à partir de l'expérience perceptive d'une conscience, expérience ellemême comprise comme le vécu phénoménal d'un vivant (ce vivant est soit biologiste soit phénoménologue) qui rencontre et perçoit un autre vivant, devenu objet de connaissance. Comme l'enfant qui «comprend le sens joyeux d'un sourire longtemps avant d'avoir vu son propre sourire ${ }^{7} »$, nous saisissons le sens d'un comportement vital. Le vital nous apparaît sous la forme significative d'un dynamisme; il renvoie, chez le vivant devenu sujet de connaissance, à un contenu de signification ${ }^{8}$. Et c'est le concept de corps propre qui prend cette "signification ${ }^{9}$ " chez Merleau-Ponty. Mais si l'organisme dont s'occupe la biologie est une telle « unité idéale ${ }^{10} »-$ c'est-à-dire un ensemble significatif pour une conscience - comprendre le rapport physique/vital revient à chercher, selon la formule étonnamment platonicienne de Merleau-Ponty, comment les phénomènes parcellaires "participent [à des idées] sans les contenir en eux ${ }^{11} »$. Le problème central de La Structure du comportement est en effet celui d'une " signification immanente ${ }^{12}$ » à l'être physique. Le vital est un contenu de signification dont s'imprègnent biologistes et phénoménologues quand leur apparaît le vivant, et c'est bien un phénomène (ce qui apparaît à une conscience) que l'un et l'autre cherchent au sein même des structures physiques.

5 Ce problème de l'immanence est lié à celui de l'ontogenèse, précisément parce qu'il suscite une interrogation ontologique sur les structurations vitales et psychiques. À propos de ces structurations, Merleau-Ponty remarque que l'existence d'une telle signification vitale «suggère celle d'une fonction générale d'organisation du comportement ${ }^{13}$ ", organisation qui se produit au niveau du substrat physique. Espérant dépasser les limites de la neurophysiologie d'inspiration pavlovienne, il fait valoir un point d'accord entre les conclusions de Piéron et celles de Goldstein, dont il tire l'idée suivante: la structure totale de comportement est au système nerveux parcellaire ce que le principe est à la conséquence ${ }^{14}$, ce que la signification est au signe. Plus clairement, et il y insiste, le substrat physico-chimique (cérébral) se coordonne et finit par se subordonner à un sens incompréhensible du point de vue de la physique parcellaire :

La coordination est maintenant la création d'une unité de sens qui s'exprime dans les parties juxtaposées, de certains rapports qui ne doivent rien à la matérialité des termes qu'ils unissent ${ }^{15}$. 
6 Sherrington, qui est le premier à réfléchir aux notions d'«intégration » et de " coordination ", émet l'idée, centrale pour toute la neurophysiologie après lui, selon laquelle l'apparition d'une fonction vitale ou psychique au sein du corps physiquechimique - l'intégration proprement dite - renvoie à l'élaboration progressive et de plus en plus complexe d'un réseau neuronal ${ }^{16}$. Mais d'après Merleau-Ponty et dans le sens d'un rejet radical du matérialisme réductionniste, les seules lois physiologiques du fonctionnement cérébral telles que les conçoit Sherrington (associations synaptiques, modulations chronaxiques) ne peuvent suffire à expliquer l'apparition d'une structure de comportement dont la signification est proprement vitale. Il dénonce le caractère " équivoque ${ }^{17}$ " des notions corrélatives d' "intégration " et de "coordination » neuronales et leur substitue les notions de «fonction d'ensemble» et de « redistribution ». La redistribution désigne la réorganisation d'un réseau neuronal de telle manière qu'il réalise une fonction - mais une réorganisation qui a lieu selon un principe non physique. L'analyse conduit à un genre particulier de structuration, proprement vitale et inassimilable à une simple complexification par combinaison de processus physiques.

7 Deux objections sont alors possibles, et l'une d'elles structure l'approche simondonienne. Pour ce qui est de la première, qui concerne l'accusation de vitalisme (excès inverse du physicalisme), notons seulement que Merleau-Ponty la réfute ${ }^{18}$ en rapportant la fonction d'ensemble non pas à une cause extérieure ou à un principe actif hypothétique et ayant rang d'être, mais à une structure comprise comme forme, forme elle-même comprise comme signification ou phénomène au sens kantien ${ }^{19}$. Le passage de la notion de «structure " à celle de "signification pour une conscience » constitue en fait la clef - et peut-être aussi la limite - de la méthode phénoménologique adoptée par Merleau-Ponty dans La Structure du comportement. Car ce n'est pas un discours sur l'être, sur les structures réelles que Merleau-Ponty veut tenir à l'aide de la conception fonctionnelle de Goldstein, mais un discours d'ordre descriptif. Son objet n'est rien de plus que l'appréhension subjective de comportements dont les structurations réelles, au niveau de l'organisme, sont seulement supposées irréductibles aux structurations physiques. Le vitalisme est ainsi remplacé par le biologisme. À la notion de «force vitale» selon Bichat, qui servait une ambition d'explication ontologique ${ }^{20}$, est substituée celle de "signification vitale ", dont le seul objectif est de servir de principe heuristique à la neurophysiologie à venir. Retournement assez étonnant donc, MerleauPonty fait avorter l'entreprise ontologique parce qu'il refuse à une phénoménologie purement descriptive toute prétention explicative.

8 Précisons alors le sens de ce déplacement relativement à l'accusation possible de finalisme, plus intéressante pour notre propos. Sur le plan ontologique, on pourrait voir dans la fonction organisatrice un vague équivalent de l'ergon aristotélicienne, qui désigne la fonction présupposée et déterminante (végétative, sensitive, motrice ou intellective) que l'être en puissance réalise dans son passage à l'acte. Dans ses cours au Collège de France sur le concept de Nature (1956-1958 et 1959-1960), Merleau-Ponty s'oppose de manière obstinée au finalisme. Mais dans La Structure du comportement (1942), tout se passe comme s'il se contentait d'un finalisme avorté par précaution. Certes, la « conception fonctionnelle » qu'il reprend à Goldstein subordonne la matière indéterminée à une fonction d'ensemble redistributive. La fonction d'ensemble renvoie bien au principe dont l'organisation physique est la conséquence. Mais parce qu'il refuse de tenir un discours ontologique, un discours sur l'être, parce qu'il préfère s'en 
tenir à un discours purement descriptif, il ne va pas jusqu'à faire de la fonction en question l'équivalent de l'acte chez Aristote, c'est-à-dire d'un principe d'individuation :

Rien n'oblige, précise Merleau-Ponty, à penser que le cycle des actions physicochimiques puisse se fermer à travers le phénomène de l'organisme, que

l'explication puisse rejoindre les données de la description. ${ }^{21}$

Il se refuse en fait à l'ontogenèse précisément pour éviter une prise de position hâtive sur le plan ontologique.

9 Aucune ontogenèse susceptible de rendre compte du caractère essentiel et génétique du vivant n'a sa place dans le projet phénoménologique de Merleau-Ponty, malgré sa volonté de décrire une forme spécifiquement vitale de structuration. Et si MerleauPonty avait tenu jusqu'au bout à l'idée d'une redistribution du substrat matériel selon ce qu'exigent les conditions normatives et structurales du comportement vital, alors le phénomène, appréhendé subjectivement, aurait fait plier le substrat matériel, ce qui aurait été un moyen violent de les accorder et de penser l'immanence et la genèse.

Pourquoi alors ne pas plutôt construire le phénomène, pour reprendre la formule de Bachelard, à travers une réflexion constructive sur la matière, réflexion qui permettrait en même temps d'expliquer les genèses et de penser l'essence de la vie ? Tel est, pour le dire vite, le sens du projet réaliste de Simondon.

\section{La spécificité du physique : Simondon chasseur de fantômes}

Simondon s'oppose à la perspective merleau-pontienne, et plus généralement à la phénoménologie, sur le plan ontologique et méthodologique. Sur le plan ontologique, il propose de penser les genèses et le caractère auto-génétique du vivant mais, et c'est déterminant, sans rejouer la rupture traditionnelle entre vital et physique. À propos de tradition, François Jacob montre très clairement, dans sa Logique du vivant ${ }^{22}$, que l'attitude biologique apparaît lorsque s'opère, à la fin xvIII ${ }^{e}$ siècle, le passage d'une classification des êtres à une autre. À l'ancienne classification, qui distinguait les êtres selon qu'ils appartenaient à l'un des trois règnes minéral, végétal ou animal, se substitue, sous la plume de naturalistes comme Lamarck ou Vicq d'Azir notamment, une bipartition entre êtres inertes et êtres vivants. Or, c'est le critère de l'organisation qui fonde cette nouvelle classification. La séparation est effectivement faite entre d'un côté l'inerte inorganisé, de l'autre le vivant précisément organisé parce qu'il possède un principe interne d'organisation.

Et c'est au nom de ce critère d'organisation que s'affirme ce qui est peut-être plus spécifique à l'attitude vitaliste, à savoir le rejet de toute explication physicaliste, réductionniste et continuiste de l'organisme (physicalisme et continuisme que Lamarck réassume par exemple, contre le vitalisme ${ }^{23}$, mais sans abandonner la perspective biologique). C'est-à-dire que pour le vitalisme le vivant ne peut être le résultat d'une simple complexification des processus matériels, ou le produit d'une causalité interne à la matière elle-même - d'où le renvoi à une causalité externe comme la force vitale. Dans ses Recherches physiologiques ${ }^{24}$, Bichat oppose en effet, au physicalisme ainsi qu'à la conception cartésienne d'un corps mécanique animé25, le simple constat d'une irréductibilité des propriétés des phénomènes biologiques aux propriétés des objets physiques. La variabilité et l'hétérogénéité des métabolismes végétal et animal, de la sensibilité et de la motilité animales par exemple, rompent avec l'invariabilité et 
l'homogénéité des phénomènes physiques. C'est pourquoi étudier et analyser le vivant comme on étudie et analyse le non-vivant ne conduit jamais qu'à substituer à la vie, la mort. Qui étudie les fluides et sécrétions animales comme de simples réalités physiques et chimiques prône ce que Bichat appelle une "anatomie cadavérique des fluides". Contre cette chimie de la mort, Bichat en appelle à une "chimie physiologique ${ }^{26}$ ", à une science plus appropriée, biologique au sens fort, qui ne réduise pas l'organisme au corps physique-chimique, à ses lois et aux modèles explicatifs qui s'y rapportent ${ }^{27}$.

C'est bien de cette attitude vitaliste que Merleau-Ponty hérite partiellement lorsque, sous l'influence du biologisme et de la conception fonctionnelle de Goldstein, il critique le physicalisme réductionniste et continuiste - lorsqu'il fait valoir, lui aussi en vertu de la variabilité et de l'hétérogénéité des comportements vitaux, l'irréductibilité de la norme vitale à la loi physique ${ }^{28}$. Et c'est précisément cette attitude vitaliste et cette vieille critique du physicalisme continuiste qui paraissent injustifiées à Simondon :

La coutume amène, en effet, à penser que les êtres vivants ne peuvent provenir des êtres physiques, parce qu'ils sont supérieurs à ces derniers grâce à leur organisation. Cependant, cette attitude même est la conséquence d'un postulat initial, selon lequel la nature inerte ne peut receler une organisation élevée ${ }^{29}$.

En fait, la coutume a déjà été dénoncée par Bachelard. Elle est celle des philosophes qui, faute d'actualiser leurs connaissances scientifiques pour saisir la vraie richesse du matérialisme, s'en prennent à lui comme à une cible trop facile :

Nous aurons donc à insister longuement sur l'inefficacité d'un matérialisme massif, d'un matérialisme immobilisé [...]. C'est ce matérialisme massif, ingénu, périmé qui sert de cible aux critiques trop faciles de la philosophie idéaliste.

Trop nombreux sont ainsi les philosophes qui s'exercent contre un fantôme démodé 30 .

14 Or, pour Bachelard, cette richesse du matérialisme authentique tient à une nouvelle compréhension de la matière, envisagée comme la face chimique d'un être relationnel et dynamique parce qu'énergétique. Le « matérialisme de la matière ${ }^{31}$ " pour reprendre son expression, contre le "matérialisme sans matière ${ }^{32}$ " pris pour cible par une philosophie qui a peur des fantômes, doit ainsi s'entendre comme un "matérialisme énergétique [qui] s'éclaire en posant un véritable existentialisme de l'énergie ${ }^{33} »$. C'està-dire que l'énergie désigne ce par quoi la matière vit et existe, ce par quoi l'être qu'elle constitue se structure. «L'être est, il faut dire : l'énergie est. Elle est absolument ${ }^{34}$ ». Elle est «le nœud de l'activité matérielle ${ }^{35}$ » au sens où, tout atome étant quantifiable sur le plan énergétique ${ }^{36}$ (l'atome est constitué d'un noyau, lui-même constitué de protons, de neutrons et d'un nuage électronique), la matière est le lieu d'échanges et de relations énergétiques qui modifient sa structure, comme c'est le cas, par exemple, dans les mélanges chimiques. La matière porte l'énergie, et l'énergie est le principe actif du devenir de la matière - principe actif que les vitalistes allaient chercher du côté d'une force ou d'un principe vital, d'une causalité donc extérieure à la matière. Tout changement de structure de l'être matériel est ainsi le reflet d'un changement énergétique, la relation entre éléments énergétiquement disparates, et donc matériellement hétérogènes, étant l'opérateur de cette vie énergétique et matérielle.

Inspiré par la thermodynamique, Simondon insiste lui aussi sur le rôle de l'énergie dans les structurations autonomes de la matière. Est-il pour autant l'héritier de Bachelard, dont il pourrait réinterpréter le matérialisme à l'aide de schèmes tirés d'autres domaines de la physique? La question se pose puisque, de manière étonnante, Simondon affirme ne pas connaître précisément l'œuvre de Bachelard, auteur qu'il 
qualifie même, tenons-nous bien, de simple " poète ${ }^{37}$ ». La parenté n'en n'est pas moins réelle entre les deux auteurs ${ }^{38}$. Elle concerne ici le rejet, éclairé par la physique contemporaine, de l'anti-matérialisme.

Ainsi, comble de l'opposition à Merleau-Ponty qui refusait que le corps parcellaire doive son unité de sens à la matérialité des termes coordonnés, Simondon considère que la matérialité seule fournit les conditions de genèse des structures vitales :

La vie n'est pas une substance distincte de la matière; elle suppose des processus d'intégration et de différenciation qui ne peuvent en aucune manière être donnés par autre chose que des structures physiques ${ }^{39}$.

Quoi qu'en dise le vitalisme, la matière est bel et bien animée et susceptible de s'organiser. Et son organisation ne procède pas d'une causalité qui lui est extérieure. En effet, les notions d'«intégration" et de "différenciation», que Simondon fait intervenir ici, renvoient aux deux aspects du même processus génétique dont la matière est le lieu. Elles désignent respectivement l'intériorisation d'un ordre structural et énergétique extérieur par réorganisation des structures internes, et la prise de forme active du système qui se réorganise comme structure opératoire. La différence de régime d'individuation entre physique et vital tient alors non pas à l'existence de processus de structuration différents, mais au système global et aux opérations qui engagent ces processus matériels d'intégration et de différenciation. Le vital réassume pleinement une réalité matérielle - il faut comprendre structurelle et énergétique, c'est-à-dire génétique et opératoire - qui le supporte en retour.

Sur le plan méthodologique, ce qui apparaît comme un "matérialisme énergétiste ", selon la formule de Vincent Bontems, détache Simondon d'une approche seulement descriptive et lui donne un autre point de départ que l'expérience vécue et phénoménale. Il part plutôt de la matière. Sa méthode est réaliste plutôt que phénoménologique, et ce qui est réel, c'est la relation énergétique et donc matérielle dont procède l'individuation, par intégration et différenciation.

Remarquons toutefois que parler de matérialisme à propos de Simondon pose problème, puisqu'il dit lui-même que sa doctrine «n'est point un matérialisme ${ }^{40}$ ». On pourrait considérer que resituer Simondon dans la lignée de Bachelard, au nom de l'intuition commune d'un matérialisme énergétiste, n'est qu'un double abus de commentateur. Toute la question est en fait de savoir dans quelle mesure Simondon peut effectivement se présenter, malgré ce qu'il en dit lui-même, comme un matérialiste - mais un matérialiste d'un genre nouveau, peut-être pas héritier direct de Bachelard, mais sans conteste pressenti par lui.

La réponse que nous proposons suppose de circonscrire la défiance de Simondon à l'égard du matérialisme au matérialisme désuet également critiqué par Bachelard, et d'admettre que la notion de "matérialisme » n'est pas une notion simple et univoque. Le matérialisme auquel Simondon ne s'identifie pas est structuré par la bipartition traditionnelle entre êtres vivants et inertes, fondé donc sur le critère de l'organisation dont nous parlions précédemment. C'est même précisément à la validité de ce critère que Simondon s'en prend directement lorsqu'il se dissocie du matérialisme, et qu'il désamorce l'alternative entre matérialisme et spiritualisme. Le matérialisme qui est inacceptable pour lui, c'est le réductionniste, celui qui voit dans une complexification des processus propres à la matière inerte le seul moyen d'expliquer les structurations vitales et par extention psycho-sociales. Plutôt que de s'en remettre à une telle réduction du complexe au simple, il s'agit pour lui de "supposer un enchaînement 
depuis la réalité physique jusqu'aux formes biologiques supérieures, sans établir de distinction de classes et de genres ${ }^{41} »$. Simondon prône donc une forme de continuisme entre matière et vie, mais un continuisme qui, d'une part, se veut encore compatible avec la discontinuité entre le physique et le vital, d'autre part se voit délesté d'une classification hautement discutable quant à son fondement.

21 L'irréductibilité dans la continuité tient en effet à une transformation de l'organisation, à un changement dans les modalités selon lesquelles s'opère l'individuation - et non à une simple complexification :

$\mathrm{Si}$, dès le début, on estime que la matière constitue des systèmes pourvus d'un très haut niveau d'organisation, on ne peut aussi facilement hiérarchiser vie et matière. Peut-être faut-il supposer que l'organisation se conserve mais se transforme dans le passage de la matière à la vie ${ }^{42}$.

Il nous semble donc possible d'envisager la doctrine simondonnienne comme un matérialisme "reconsidéré", au sens où elle renvoie à une conception selon laquelle le vital apparaît au sein d'une réalité entièrement et seulement matérielle, sans être pour autant réductible, dans son organisation et dans ses modes opératoires, à un système physique. Nous disons " entièrement et seulement matérielle ", dans la mesure où le régime d'individuation vitale, au sein duquel l'existence psycho-sociale et spirituelle de l'individu prend place chez Simondon, est à concevoir dans les termes d'une transformation de l'organisation d'un être toujours matériel, non comme le passage d'une substance à une autre. En quelque sorte, dire que tout est matériel chez Simondon signifie que, dans sa conception, la matière peut être tout ce qu'elle n'est pas dans un matérialisme traditionnel et réductionniste - ou encore, tout ce qu'elle n'est pas seulement dans un simple système d'individuation physique. C'est en ce sens fort que le matérialisme de Simondon est "reconsidéré ». Il faut donc, pour justifier cette qualification qui est la nôtre, commencer par admettre que la notion de "matérialisme » n'est pas simple et repérer ne serait-ce qu'un matérialisme non réductionniste par opposition au matérialisme réductionniste et traditionnel. Tout l'enjeu est alors de savoir ce qui caractérise, au-delà de son aspect énergétiste, le matérialisme de Simondon, et dans quelle mesure ce matérialisme permet effectivement de penser le caractère autogénétique du vivant et, ce faisant, la normativité vitale.

\section{Matérialisme néoténique, affectivité et normativité}

Continuisme ne signifie donc pas réductionnisme chez Simondon. La perspective continuiste, Simondon la fait justement valoir contre la conception fonctionnelle de Goldstein que réassume Merleau-Ponty :

Il est regrettable que la systématique holistique du biologisme, telle qu'elle est présentée par Goldstein, soit conçue comme nécessairement macrophysique, prise sur la totalité d'un organisme complexe. L'ontologie parménidienne de Goldstein empêche tout rapport entre l'étude du vivant et l'étude de l'inerte, dont les processus sont microphysiques. Il peut $\mathrm{y}$ avoir un ordre intermédiaire de phénomènes, entre le microphysique parcellaire et l'unité organismique macrophysique ; cet ordre serait celui des processus génétiques, chronologiques et topologiques, c'est-à-dire des processus d'individuation, communs à tous les ordres de réalité en lesquels s'opère une ontogénèse ${ }^{43}$. 
24 proprement vital mais radicalement rapporté à sa matérialité, conduit Simondon à une réflexion ontologique sur ce qu'il nomme le " préindividuel », c'est-à-dire sur l'être des disparations (ou incompatibilités) structurales et énergétiques qui se résolvent et qui sont au principe de toute individuation, de toute prise de forme. Contre l'ontologie parménidienne, Simondon opère en quelque sorte un retour à l'ontologie héraclitéenne. Il envisage une individuation à partir des disparations de l'être. Le préindividuel renvoie ainsi à l'ensemble des conditions hétérogènes sur le plan énergétique et structural, constitutives de l'être, et qui sont aussi bien présupposées que contemporaines de l'individuation. Le préindividuel est donc relationnel et, en tant que tel, «métastable ». L'état métastable (notion héritée de la thermodynamique), ni stable ni purement instable, est bien celui d'une relation riche en potentiels issus de la disparation même et initiateurs du changement énergétique et structural (l'individuation).

La matière est ainsi le support commun aux deux régimes d'individuation physique et vital. En ce qui concerne la différence entre physique et vital, Jean-Hugues Barthélémy résume parfaitement les choses lorsqu'il écrit que «la différence entre l'individu physique et l'individu vivant est alors que le second entretient en lui une métastabilité, tandis que le premier est devenu stable et a épuisé ses potentiels ${ }^{44} »$. Nous n'entrerons pas ici dans le détail du passage tel que le pense Simondon, du régime d'individuation physique au régime vital. Retenons seulement que la notion de "néoténie », à laquelle Barthélémy renvoie directement, permet de retrouver, au sein de ce qui ne peut être un simple continuisme matérialiste, une discontinuité entre les deux ordres de réalité. L'hypothèse de Simondon est que " le vital serait du physique en suspens ${ }^{45}$ ", au sens où l'individuation vitale «retient et dilate la phase la plus précoce de l'individuation physique $^{46}{ }^{\prime}$. C'est-à-dire que plutôt que d'épuiser ses potentiels dans la réalisation d'une structure définitive et stable, le vivant conserve en lui quelque chose de la tension préindividuelle dont procèdent les structurations.

Au fond, le sens que Simondon donne à ce terme de "néoténie » est proche de son étymologie, puisqu'il est obtenu à la fin du $\mathrm{xIX}^{\mathrm{e}}$ siècle $^{47}$ par la juxtaposition du préfixe grec neo qui signifie «nouveau », et du verbe teinein, signifiant «étendre ». Le vivant conserve un pouvoir de structuration, une " capacité de transduction, grâce à laquelle [les espèces biologiques] peuvent s'étendre indéfiniment ${ }^{48}{ }^{4}$. Cette capacité caractérise le système d'individuation vitale par contraste d'avec le système physique.

Or, c'est l'affectivité qui entretient la métastabilité dans le vivant. L'affectivité est en effet le système de transduction, mais un système qui s'entretient lui-même comme capacité de transduction, comme pouvoir de polarisation et de structuration :

La transduction est opérée par l'affectivité et par tous les systèmes qui jouent dans l'organisme le rôle de transducteurs à divers niveaux. L'individu serait donc toujours un système de transduction, mais, alors que cette transduction est directe et à un seul niveau dans le système physique, elle est indirecte et hiérarchisée dans l'être vivant. ${ }^{49}$

La transduction désigne, chez Simondon, le processus de structuration du réel par lequel s'opère l'individuation. Elle consiste en la propagation d'une activité de proche en proche, par structuration progressive d'un domaine de réalité. Toute individuation s'entend ainsi comme information ou prise de forme, par transduction. Or, si l'affectivité est comprise par Simondon comme l'être de la relation individuante entre 
intégration et différenciation, toute intégration et toute différenciation constituent une réorganisation structurale et énergétique du système, réorganisation dont l'effet est un approfondissement de la réalité préindividuelle, un renouvellement ou un enrichissement du problème dont procède, comme solution appelée, l'individuation. En d'autres termes, ce sont les disparations dont procèdent l'individuation et, avec elles, la problématique interne du système, qui se renouvellent sous l'effet de l'intégration et de la différenciation. Si conservation du pouvoir de structuration il y a, c'est au sens où toute nouvelle intégration et toute nouvelle différenciation s'accompagnent d'une nouvelle potentialisation.

Il faudrait insister davantage sur cette notion d'affectivité, absolument centrale dans la pensée simondonienne du vivant - notamment sur le rôle synthétique et de polarisation des qualités affectives, qui permettent aux produits élaborés de l'activité intégratrice et de l'activité différenciatrice de se cohérer au sein d'une identité et d'une unité du sujet ${ }^{50}$. C'est à travers cette notion que Simondon propose de traiter le problème de la relation psychophysiologique, qui l'intéressait originellement. Contentons-nous de souligner que cette conception rompt avec l'ontologie parménidienne selon laquelle, s'il y a genèse, c'est à partir du même. Si l'on en croit le Poème de Parménide, « de ce qui est », on ne peut «faire naître quelque autre chose ${ }^{51}$ ». Contre cette affirmation, contre cet autre poète, Simondon propose d'envisager l'engendrement $\mathrm{du}$ vital au sein $\mathrm{du}$ physique - l'engendrement avec changement d'ordre de réalité étant bien contraire à l'ontologie parménidienne. Enfin, chez Goldstein les équilibres préexistent en l'organisme et la question de l'avènement des normes comportementales qui les réalisent n'est pas posée. Toute rééquilibration dans l'adaptation à un milieu perturbateur consiste en un rétablissement du même équilibre. Le nouvel équilibre advient pour autant que, pour pouvoir se rétablir, il préexiste au moins virtuellement dans l'organisme, comme ensemble de conditions physiologiques requises. Tel est le sens de ces " conditions virtuelles ${ }^{52}$ » en vertu desquelles MerleauPonty fait valoir la spécificité des comportements vitaux par rapport aux phénomènes physiques dans $L a$ Structure $d u$ comportement. Mais ces conditions virtuelles, physiologiques (on pense aux différents équilibres homéostasiques) ne permettent pas encore de penser la structuration des normes comportementales et individuelles à travers lesquelles elles se rétablissent. Substituer comme le fait Simondon la notion de "potentiel réel » à celle de "virtuel ", c'est se donner un moyen conceptuel pour penser la normativité. Plus qu'une simple application de normes préconstituées, la vie s'entend justement, chez Canguilhem, comme un mouvement d'élaboration spontanée et progressive de normes. La normativité, qui caractérise essentiellement la vie selon lui, désigne un " pouvoir de révision et d'institution des normes ${ }^{53}$ ", un « certain jeu des normes de la vie et du comportement ${ }^{54} »$. Reprenons la formule de Pierre Macherey :

Parler de normativité, c'est bien, au lieu de considérer la mise en œuvre des normes comme l'application mécanique d'un pouvoir préconstitué, montrer comment le mouvement concret des normes, qui sont des schèmes vitaux à la recherche des conditions de leur réalisation, élabore au fur et à mesure de son déroulement ce pouvoir qu'il produit à la fois sur le plan de sa forme et de son contenu ${ }^{55}$.

C'est bien un tel pouvoir de structuration, une telle normativité saisie au niveau de l'individu, plus précisément du sujet - et non à celui de l'espèce -, que Simondon permet de penser. La normativité ne renvoie alors pas à la présence de constantes biologiques ou au caractère structuré des comportements collectifs ou individuels. Elle indique davantage l'existence d'un pouvoir d'élaboration des normes. Canguilhem a 
déjà suffisamment insisté - et Simondon, pour le coup, ne l'ignore pas -, sur le fait que plus que des êtres normés, nous sommes des êtres normatifs ${ }^{56}$. Il faut que les normes " craquent $^{57}$ » et se renouvellent, pour reprendre sa formule, que nous soyons nousmêmes ce craquage et ce renouvellement, êtres vivants à proprement parler.

L'ontologie du préindividuel pourrait bien être un moyen, pour Simondon, de poser les fondements de ce que Canguilhem a toujours refusé, à savoir une biologie de philosophe qui rende compte, au-delà du dynamisme préconstitué et à la place du biologiste, des soubassements ontologiques de la normativité vitale ${ }^{58}$. Mais il faut être un peu plus précis et remarquer que ce qui distingue Simondon de Merleau-Ponty, c'est un rapport tout à fait particulier à la biologie. Là où le premier Merleau-Ponty opère un retour en arrière, un retour aux origines de l'attitude biologique, s'abreuvant un peu et bien malgré lui, dans La Structure du comportement, au sein d'une tradition vitaliste, Simondon se contente de proposer des hypothèses directrices elles-mêmes inspirées des sciences physiques et biologiques. Pour reprendre l'analyse de Jean-Hugues Barthélémy, « dire que l'épistémologie est requise par l'ontologie, c'est reconnaître que Simondon prête aux siences, et plus précisément à la physique contemporaine, ce que Bachelard, parlant de la relativité einsteinienne, nommait une "valeur inductive ». Par là, il ne faut pas entendre une capacité de la théorie physique à se construire par induction, mais une portée philosophique qui autorise l'ontologie philosophique à se construire à partir de ce que lui enseigne la physique ${ }^{59}$ ". Il ne s'agit donc pas, pour Simondon, de substituer une biologie philosophique à une biologie scientifique, mais d'enrichir mutuellement biologie et ontologie philosophique, admettant que les hypothèses construites philosophiquement à partir de la science, peuvent fournir à cette dernière quelques pistes de recherche.

\section{Conclusion}

On ne peut pas marier l'un à l'autre le discours phénoménologique et le discours réaliste de Simondon. Une telle tentative revient à ignorer la différence radicale entre deux registres de discours, le premier portant sur les données subjectives et le procès de constitution du sujet transcendantal, l'autre sur la réalité des processus physiques. Les deux projets sont incompatibles, aussi bien au niveau des partis pris épistémologiques et ontologiques qui les structurent, que des méthodes et des fins qui les régissent. Faire dialoguer Merleau-Ponty ou, plus généralement, la phénoménologie avec Simondon ne peut ainsi avoir qu'une fonction: circonscrire toujours davantage cette différence épistémologique et ontologique.

S'intéresser au problème d'une conception adéquate de l'essence de la vie suffit à s'en convaincre. Traditionnellement, on veut penser la vie à travers un rejet du matérialisme, à travers un antiphysicalisme facile et désuet. Or, la force de Simondon, c'est de montrer qu'on ne pense la vie que dans un matérialisme reconsidéré. En effet, penser le caractère autogénétique du vivant revient, chez Simondon, à chercher les conditions ontologiques matérielles de l'individuation, à réfléchir aux modifications partielles des modes de l'individuation physique qui marquent l'avènement d'un système vital et autogénétique. Les deux écueils de la méthode phénoménologique sont ainsi évités, qui consistaient d'une part à orienter la réflexion vers une simple description, incapable de rendre compte de ce que doit être le système de structuration vitale pour pouvoir être porteur et créateur de normes, et d'autre part à ne pas 
interroger authentiquement le lien de continuité entre les ordres de réalité physique et vital. De Merleau-Ponty à Simondon, on passe ainsi d'une simple appréhension subjective du comportement vital, qui laisse sans solution le problème de l'immanence de la signification vitale au physique, à une ontogenèse susceptible, quoi que de manière purement hypothétique, de montrer aussi bien la continuité que la discontinuité entre le physique et le vital. Pour reprendre la formule étonnante de Bachelard, la matière "existe " parce qu'elle est le lieu d'échanges énergétiques, et c'est cette riche existence qui explique in fine le caractère auto-génétique du vivant, et l'intégration non réductrice du vital au physique.

\section{BIBLIOGRAPHIE}

Barthélémy Jean-Hugues (dir.), Cahiers Simondon, numéro 6, Paris, L'Harmattan, 2015.

Bichat Marie François Xavier, Recherches physiologiques sur la vie et la mort, Victor Masson, Paris, 1859 [1800].

Bichat François Xavier, Recherches sur le fluide nerveux, in Recherches sur l'organisation des corps vivants, Paris, Maillard, 1802.

Bontems Vincent, «Quelques éléments pour une épistémologie des relations d'échelle chez Gilbert Simondon », Appareil (revue en ligne), 2, 2008 (http://appareil.revues.org/595).

Canguilhem Georges, La Connaissance de la vie, Paris, Vrin, 2009.

Canguilhem Georges, Le Normal et le pathologique, Vendôme, Puf, 1972.

Canguilhem Georges, La Formation du concept de réflexe, aux XVII ${ }^{e ̀ m e}$ et XVIII ${ }^{e}$ siècles, Mayenne, Vrin, 1977 [1955].

Dufrenne Mikel, Revue de Métaphysique et de Morale, « Un livre récent sur la connaissance de la vie », 1953, 1/2 (janvier/juin 1953).

Goldstein Kurt, La Structure de l'organisme, Paris, Gallimard, 1951 [1934].

Jacob François, La Logique du vivant, Saint Armand-Montrond, Gallimard, 1995 [1976].

Macherey Pierre, « Normes vitales et normes sociales dans L'Essai sur quelques problèmes concernant le normal et le pathologique », in Actualité de Georges Canguilhem - Le normal et le pathologique. Actes $\mathrm{du} \mathrm{X}^{\text {ème }}$ colloque de la société internationale d'histoire de la psychiatrie et de la psychanalyse, éditions de l'Institut Synthélabo pour le progrès de la connaissance, collection Les Empêcheurs de penser en rond, 1998.

Merleau-Ponty Maurice, La Structure du comportement, Paris, Puf, 2009 [1942].

Sherrington Richard Scott, The Integrative Action of the Nervous System, New York, Charles Scribner's Sons, 1906.

Simondon Gilbert, L'individuation à la lumière des notions de forme et d'information, Grenoble, Éditions Jérôme Millon, 2013 [1958]. 
Simondon Gilbert « Entretien sur la mécanologie : Gilbert Simondon et Jean Le Moyne », in Sur la technique, Paris, Puf, 2014.

\section{NOTES}

1.

Le terme s'applique aujourd'hui davantage aux végétaux. Il est remplacé, à propos des animaux, par le terme de « taxie».

2. Kurt Goldstein, La structure de l'organisme, Paris, Gallimard, 1951 (1934), VII.

3. «Un livre récent sur la connaissance de la vie » in Revue de Métaphysique et de Morale, 1953 (1/2), janvier/juin 1953, p. 181.

4. Nous avions confronté une première fois Merleau-Ponty et Simondon dans un ouvrage collectif dirigé par Jean-Hugues Barthélémy, Cahiers Simondon, numéro 6, « De Merleau-Ponty à Simondon, la valeur épistémologique de la notion de norme », Paris, L’Harmattan, 2015, p. 131-144.

5. Gilbert Simondon, L'individuation à la lumière des notions de forme et d'information (désormais noté ILFI), Grenoble, Éditions Jérôme Millon, 2013 [1958].

6. Vincent Bontems, "Quelques éléments pour une épistémologie des relations d'échelle chez Gilbert Simondon », Appareil (revue en ligne), 2, 2008 http://appareil.revues.org/595.

7. Maurice Merleau-Ponty, La Structure du comportement (désormais noté SC), Paris, Puf, 1942, p. 169.

8. En ce sens « la connaissance, au lieu d'être la présentation au sujet d'un tableau inerte, est l'appréhension du sens de ce tableau [...]. Toute chose à laquelle on puisse penser est une signification de chose ». Ibid., p. 215.

9. Dans cette perspective, la frontière entre objet et sujet est brouillée sous l'effet syncrétique de la notion de vivant. Aussi le concept de corps propre désigne-t-il également cela qui perçoit, que vivant connaissant et vivant connu partagent.

10. Merleau-Ponty, La Structure du comportement, op. cit., p. 165.

11. Id.

12. Ibid., p. 170 .

13. Ibid., p. 74.

14. Ibid., p. 70.

15. Ibid., p. 96. Nous soulignons.

16. Richard Scott Sherrington, The Integrative Action of the Nervous System, New York, Charles Scribner's Sons, 1906.

17. Merleau-Ponty, La Structure du comportement, op.cit., p. 84.

18. Ibid., p. 166-173.

19. Id.

20. À ce propos, on peut se reporter à François Jacob, La Logique du vivant, II, «La vie ", Saint Armand-Montrond, Gallimard, 1995 [1976], p. 106.

21. Merleau-Ponty, La Structure du comportement, op.cit., p. 172.

22. François Jacob, La Logique du vivant, op. cit., p. 100.

23. Voir sur ce point ses Recherches sur le fluide nerveux, in Recherches sur l'organisation des corps vivants, Paris, Maillard, 1802, p. 189 et suivantes.

24. Marie François Xavier. Bichat Recherches physiologiques sur la vie et la mort, I « Recherches sur la vie », article 7 « Différences générales des deux vies, par rapport aux forces vitales », réédition, Victor Masson, Paris, 1859 (1800), p. 55-82.

25. Contre Descartes et contre toute forme d'animisme, Bichat fait aussi valoir l'inhérence des processus affectifs, sentimentaux et intellectuels à la vitalité animale. Là où l'animisme pense la 
vie et la matière comme subordonnées à une causalité spirituelle, le vitalisme décrit une subordination des ordres matériel et spirituel au vital.

26. Ibid., p. 58.

27. Voir Ibid., p. 58-59 : « Il est facile de voir, d'après cela, que la science des corps organisés doit être traitée d'une manière toute différente de celles qui ont les corps inorganiques pour objet. Il faudrait, pour ainsi dire, y employer un langage différent; car la plupart des mots que nous transportons des sciences physiques dans celles de l'économie animale ou végétale, nous y rappellent sans cesse des idées qui ne s'allient nullement avec les phénomènes de cette science ».

28. Voir Merleau-Ponty, La Structure du comportement, op.cit., p. 161.

29. Simondon, «Information et ontogenèse : l'individuation vitale », ILFI, op.cit., p. 159.

30. Gaston Bachelard, « Introduction », Le Matérialisme rationnel, Paris, Puf, 2014 [1953], p. 3.

31. Id.

32. Ibid., p. 4.

33. Ibid., p. 177.

34. Id.

35. Ibid., p. 182.

36. On peut à ce propos renvoyer à Bachelard, Le Nouvel esprit scientifique, Paris, Puf, 1991 [1934], p. 82 et suivantes.

37. Simondon, «Entretien sur la mécanologie: Gilbert Simondon et Jean Le Moyne », Sur la technique, Paris, Puf, 2014, p. 415.

38. Sur cette question de la parenté entre la conception simondonienne et le réalisme des relations chez Bachelard, voir J.-H. Barthélémy, "Le "réalisme des relations" un préalable ontologique », Simondon ou l'encyclopédisme génétique, Paris, Puf, 2008.

39. Simondon, ILFI, op. cit., p. 162.

40. Ibid., p. 158.

41. Id.

42. Id.

43. Ibid., II " individuation et information ", 2 "information et ontogenèse ", 5 «topologie et ontogenèse ", p. 229.

44. Jean-Hugues Barthélémy, "glossaire Simondon", sur la notion de métastabilité, Cahiers Simondon, numéro 5, Paris, L'Harmattan, 2013, p. 122.

45. Simondon, ILFI, op. cit., p. 152.

46. Ibid.

47. Cf. Julius Kollmann (1885).

48. Simondon, ILFI, op.cit., p. 161. Nous soulignons.

49. Ibid.

50. Sur ce point, cf. Ibid., p. 162.

51. Parménide, Poème, VIII, trad. Tannery.

52. Merleau-Ponty, La Structure du comportement, op.cit., p. 157.

53. Georges Canguilhem, «Le Normal et le pathologique », La Connaissance de la vie, Paris, Vrin, 2009, p. 217.

54. Ibid. p. 215.

55. Pierre Macherey, "Normes vitales et normes sociales dans L'Essai sur quelques problèmes concernant le normal et le pathologique", in Actualité de Georges Canguilhem - Le normal et le pathologique. Actes du $X^{e}$ colloque de la société internationale d'histoire de la psychiatrie et de la psychanalyse, Institut Synthélabo pour le progrès de la connaissance, coll. «Les Empêcheurs de penser en rond ", 1998, p. 74.

56. Georges Canguilhem, "Norme et moyenne », Le Normal et le pathologique, II, 3 Vendôme, Puf, 1972, p. 106.

57. Id. 
58. Georges Canguilhem, " avant-propos », La Formation du concept de réflexe, aux XVII et XVIII ${ }^{e}$ siècles , Mayenne, Vrin, 1977 [1955], p.1. Canguilhem parle d'une «biologie de philosophe, projet monstrueux ».

59. J.-H. Barthélémy, "Le "réalisme des relations", un préalable ontologique", Simondon ou l'Encyclopédisme génétique I, Paris, Puf, 2008, p. 9.

\section{RÉSUMÉS}

Dégager le contexte philosophique et scientifique dans lequel la pensée de Simondon s'inscrit permet d'en saisir toute la force et toute l'originalité. Dès 1942, Merleau-Ponty réinvestit le problème de la relation psycho-physiologique qu'il prétend traiter dans les termes d'un antiphysicalisme traditionnel avec lequel Simondon veut rompre. Tout l'enjeu pour Simondon, qui s'intéresse à ce problème à partir de 1952, est de concilier le matérialisme avec la reconnaissance d'une spécificité du fait vital et du fait humain, en d'autres termes, de proposer un matérialisme non réductionniste. C'est dans ce cadre que la notion de vie devient centrale et que le problème sous-jacent de la spécificité du vital par rapport au physique prend toute son importance. Penser l'essence de la vie sans la détacher complètement de sa dimension matérielle exige une intense réflexion ontologique et épistémologique, à l'aune de laquelle seulement le traitement de la relation psycho-physiologique devient possible. Il s'agira ici de montrer comment, face au biologisme goldsteinnien et à la phénoménologie merleau-pontienne qui en hérite directement, la perspective matérialiste de Simondon est révolutionnaire.

\section{INDEX}

Mots-clés : vie, matérialisme, phénoménologie

\section{AUTEUR}

\section{ÉMILIEN DERECLENNE}

Doctorant en philosophie de l'esprit et sciences cognitives à l'université Paris 1 Panthéon Sorbonne. Professeur de philosophie emiliendereclenn@aol.com 